\title{
Portable Gait Lab: Zero Moment Point for Minimal Sensing of Gait*
}

\author{
Mohamed Irfan Mohamed Refai ${ }^{a, 1}$, Bert-Jan F. van Beijnum ${ }^{1}$, Jaap H. Buurke ${ }^{1,3}$, Mique Saes ${ }^{2}$, \\ Johannes B.J. Bussmann ${ }^{4}$, Carel G. Meskers ${ }^{2}$, Erwin van Wegen ${ }^{2}$, Gert Kwakkel ${ }^{2}$, and Peter H. Veltink ${ }^{1}$
}

\begin{abstract}
Ambulatory sensing of gait kinematics using inertial measurement units (IMUs) usually uses sensor fusion filters. These algorithms require measurement updates to reduce drift between segments. A full body IMU suit can use biomechanical relations between body segments to solve this. However, when minimising the sensor set, we lose a lot of this information. In this study, we explore the assumptions of zero moment point (ZMP) as a possible source of measurement updates for the sensor fusion filters. ZMP is otherwise utilised for humanoid gait in robots. In this study, first, the relation between the ZMP and centre of pressure $(\mathrm{CoP})$ is studied using a GRAIL system, consisting of opto-kinetic measurements. We find that the mean distance over the gait cycle between $\mathrm{ZMP}$ and $\mathrm{CoP}$ is $10.5 \pm 1.2 \%$ of the foot length. Following this, we show how these results could be used to improve measurements in a minimal IMU based sensing setup.
\end{abstract}

\section{INTRODUCTION}

Ambulatory estimation of gait measures is useful in understanding gait patterns in healthy subjects, and also recovery in people with gait impairment [1]. One possible ambulatory method is to use Inertial Measurement Units (IMUs). IMUs consists of 3D accelerometers, and 3D gyroscopes, and are small and wearable. They can be used to estimate full body kinematics, and also kinetics, if a full body suit of IMUs is used [2], [3].

IMUs have also been widely explored for minimal sensing of gait [4]. Several algorithms including machine learning and sensor fusion approaches have been applied in order to estimate spatial and temporal parameters from a small set of IMUs [5], [6]. Sensor fusion approaches derive movement velocity and position of the segment they are attached to. However, they are affected by drift. Additionally, the IMUs do not have a sense of relative distance between each segment. In a full body setup, biomechanical constraints are used to solve this issue [2]. However, in a minimal sensing setup, such as IMUs on the feet, this may cause the two feet to drift apart from each other. This issue has been solved either as a problem with inequality constraints [7], or using biomechanical constraints based on the inverted pendulum model of human motion [6].

The theory of Zero moment point (ZMP) has been extensively used to balance gait in humanoid systems [8]. It

\footnotetext{
*This work is part of the Perspectief programme NeuroCIMT with project number 14905 which is (partly) financed by the Netherlands Organisation for Scientific Research (NWO).

$a_{\text {m. }}$ i.mohamedrefaidutwente.nl

${ }^{1}$ Biomedical Signals and Systems, Universiteit Twente, Enschede, The Netherlands

${ }^{2}$ Amsterdam University Medical Center, Amsterdam, The Netherlands

${ }^{3}$ Roessingh Research and Development, Enschede, The Netherlands

${ }^{4}$ Erasmus MC University Medical Center, Rotterdam, The Netherlands
}
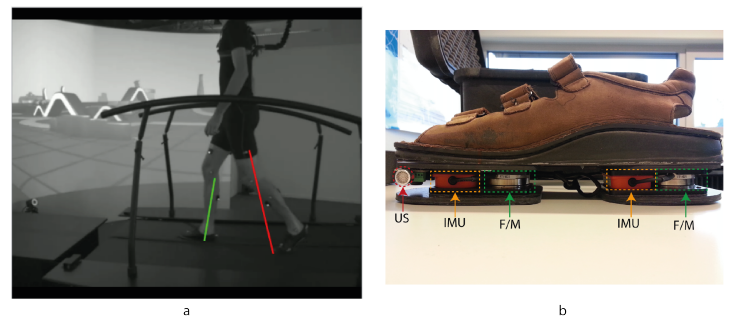

Fig. 1. Measurement setups used in this study. (a) is the GRAIL platform and (b) is the ForceShoe ${ }^{\mathrm{TM}}$

assumes that for a stable gait pattern, the moments around the centre of mass $(\mathrm{CoM})$ are zero. Solving this assumption, we can derive relations between ZMP, CoM, and distances between the feet. This could be potential information that would reduce drift in the sensor fusion approaches. Therefore, in this study, we explore the assumptions of ZMP to identify biomechanical constraints that would be useful as a measurement update for sensor fusion filters. First, we study the differences between ZMP and the reference center of pressure $(\mathrm{CoP})$ for walking in two conditions; normal and a casted condition. This is measured in an opto-electronic setup. Using the same setup, we test a relation derived from the ZMP that could provide relative distances between each foot and CoM. Further, we show an IMU example to describe steps to implement the ZMP in IMU based sensor fusion approaches.

\section{METHOD}

\section{A. Measurement Setup}

Seven healthy female subjects were asked to walk on the GRAIL (Motekforce Link, The Netherlands). As can be seen in Fig. 1 (a), the GRAIL consists of a split belt treadmill with force plates and ten VICON motion capture cameras to collect gait biomechanics. The setup measured the 3D ground reaction forces and also the 3D kinematics of the body positions. The subjects' average age was $22.9 \pm 1.4$ years, height was $1.78 \pm 0.06 \mathrm{~m}$, and weight was $73.4 \pm 5.4$ $\mathrm{kg}$. The subjects were asked to walk for 5 minutes on the treadmill at $1.2 \mathrm{~m} / \mathrm{s}$. After this, a plaster technician casted the right foot of the subject and they were asked to walk again for 5 minutes at the same speed on the treadmill. Casting was done to induce asymmetry in gait. The institutional ethical review board of the Vrij University Amsterdam approved the experimental procedure in this study. All subjects provided written informed consent. 


\section{B. Centre of Mass}

Schepers et al. [9] used a complementary filter algorithm to estimate low and high frequency components of CoM from the $3 \mathrm{D}$ forces and moments measured from the ForceShoe ${ }^{\mathrm{TM}}$ (Fig. 1 (b)). Here, we apply the algorithms to measurements from the GRAIL, as it has been validated against the CoM estimations using segmental kinematics [9]. Additionally, as we use the ForceShoe ${ }^{\mathrm{TM}}$ in the later part of the study, the same algorithms are applied here. The first stage estimates CoM from both foot kinetic and kinematic information by low pass filtering the CoP to estimate the position of CoM. The total body CoP is estimated as follows from the force measurements on the GRAIL.

$$
C o P_{a x}=\frac{F_{Z, l} \cdot C o P_{a x, l}}{F_{Z, l}+F_{Z, r}}+\frac{F_{Z, r} \cdot C o P_{a x, r}}{F_{Z, l}+F_{Z, r}}
$$

All variables in (1) are a function of time. Equation (1) is depicted in the global frame with $\mathrm{Z}$ axis positive upwards along the vertical, and $\mathrm{X}$ positive along the walking direction. Here, subscript $r$ and $l$ stand for the right and left foot respectively, and subscript $a x$ corresponds to either $\mathrm{X}$ or $\mathrm{Y}$ axes. $F_{z}$ refers to the force in the $\mathrm{Z}$ axis. The $C_{o} P_{a x}$ is then low pass filtered at $0.4 \mathrm{~Hz}$ to obtain the $C o M_{a x, L F}$ [9].

The second algorithm estimates CoM from kinetic information alone by double integration of the net forces based on Newton's second law. The acceleration of the body mass $m_{\text {body }}$ at the CoM is given as follows.

$$
\mathbf{a}_{C o M}=\frac{\mathbf{F}}{m_{b o d y}}+\mathbf{g}
$$

Here, $\mathbf{F}$ is the net force vector acting on the body, and $\mathbf{g}$ is the gravitational acceleration. The change in CoM position over time was derived from integrating the $\mathbf{a}_{C o M}$ twice. This results in $\mathbf{x}_{C o M}$ which was high pass filtered with a cut off at $0.4 \mathrm{~Hz}$ to obtain $C o M_{a x, H F}$. This is the same cut off as that of $C o M_{a x, L F}$ 's low pass filter. The $C o M_{a x, L F}$ and $C o M_{a x, H F}$ are fused using a complementary filter to obtain the trajectory of CoM.

\section{Zero Moment Point}

The theory of ZMP assumes that the moment around the CoM is zero for a stable walking trajectory. Here, ZMP is a point on the ground, such that the cross product of the vector (r) joining the CoM and ZMP and the ground reaction force vector $(\mathbf{F})$ is zero. This gives us the following equations:

$$
\begin{gathered}
\mathbf{r} \times \mathbf{F}=0 \\
(C o M-Z M P)_{a x} \cdot F_{Z}=(C o M-Z M P)_{z} \cdot F_{a x} \\
Z M P_{a x}=C o M_{a x}-\left(r_{Z} \cdot \frac{F_{a x}}{F_{Z}}\right)
\end{gathered}
$$

In (4), $Z M P_{Z}$ is zero as it lies on the floor, and $r_{Z}$ is estimated as height of pelvis from the GRAIL system. Therefore, (5) provides ZMP positions in $\mathrm{X}$ and $\mathrm{Y}$ axes. This is then compared with the $\mathrm{CoP}$ estimated from the treadmill force plates using (1).

\section{Application of Zero Moment Point}

The relation between ZMP and CoM as shown in (5) can be utilised as additional information about relative distance between the feet and CoM. Therefore, they can be used as measurement updates for a sensor fusion filter, if the other variables are known. For example, during swing phase of the left foot, the CoP of the body will lie under the right foot. Without pressure insoles, it is not straight forward to measure CoP of each foot. However, the foot positions can be estimated using an IMU on each foot [10], and CoM can be tracked using a pelvis IMU [11]. Here, we can provide an estimate for the right foot during left foot swing phase as:

$$
\operatorname{pos}_{a x, r}=C o M_{a x, s l}-\left(r_{Z} \cdot \frac{F_{a x}}{F_{Z}}\right)
$$

Here, $\operatorname{pos}_{a x, r}$ is position of the right foot, and subscript $s l$ denotes instances of left foot swing phase. We have assumed that the differences in ZMP and foot position is trivial. Subsequently, we can derive an estimate for the left foot during the swing phase of the right foot $(s r)$.

$$
\operatorname{pos}_{a x, l}=C o M_{a x, s r}-\left(r_{Z} \cdot \frac{F_{a x}}{F_{Z}}\right)
$$

We then compare $\operatorname{pos}_{a x, r}$ and $\operatorname{pos}_{a x, l}$ with the true foot positions at the respective instances ( $s l$ and $s r$ ).

\section{E. Example using IMUs}

We show a possible application of (6) and (7) in practice by describing a preliminary measurement. A subject (male, $71 \mathrm{~kg}, 1.78 \mathrm{~m}$ tall, and 25 years old) is asked to walk with the ForceShoes $^{\mathrm{TM}}$ for $10 \mathrm{~m}$ in a straight line. There is an IMU on each foot. We apply the sensor fusion filter of Weenk et al [10] to the measurements by the IMU. This includes their prediction models, and measurement updates such as zero velocity update, and zero height update. However, we skip the measurement updates from the ultrasound sensor, as this includes relative foot distance information. Therefore, we obtain foot positions, $\operatorname{pos}_{a x, r}^{w k}$ and $\operatorname{pos}_{a x, l}^{w k}$. These will have drifted due to noise, due to absence of any relative distance information. Then, we estimate reference CoM trajectory using forces measured by the ForceShoes ${ }^{\mathrm{TM}}$ [9]. Further, we apply (6) and (7) to the CoM estimated, in order to estimate $\operatorname{pos}_{a x, r}^{z m}$ and $\operatorname{pos}_{a x, l}^{z m}$, respectively. Here, we assume $r_{z}$ is a constant line, with a value equal to the subject's pelvis height during quiet standing. We plot the trajectories of interesting parameters and comment on how this could be used in a sensor fusion setup.

\section{F. Analysis of Results}

We compare the $Z M P_{a x}$ measured in (5) with the CoP measured from the treadmill force plates in GRAIL. Then, we compare the error as a percentage of subject's foot length $\beta \%$ with Herr et al [12]. Following this, we compare $\operatorname{pos}_{a x, r}$ and $\operatorname{pos}_{a x, l}$ from (6) and (7) with foot positions measured by VICON in GRAIL. For each of these, we test if the 


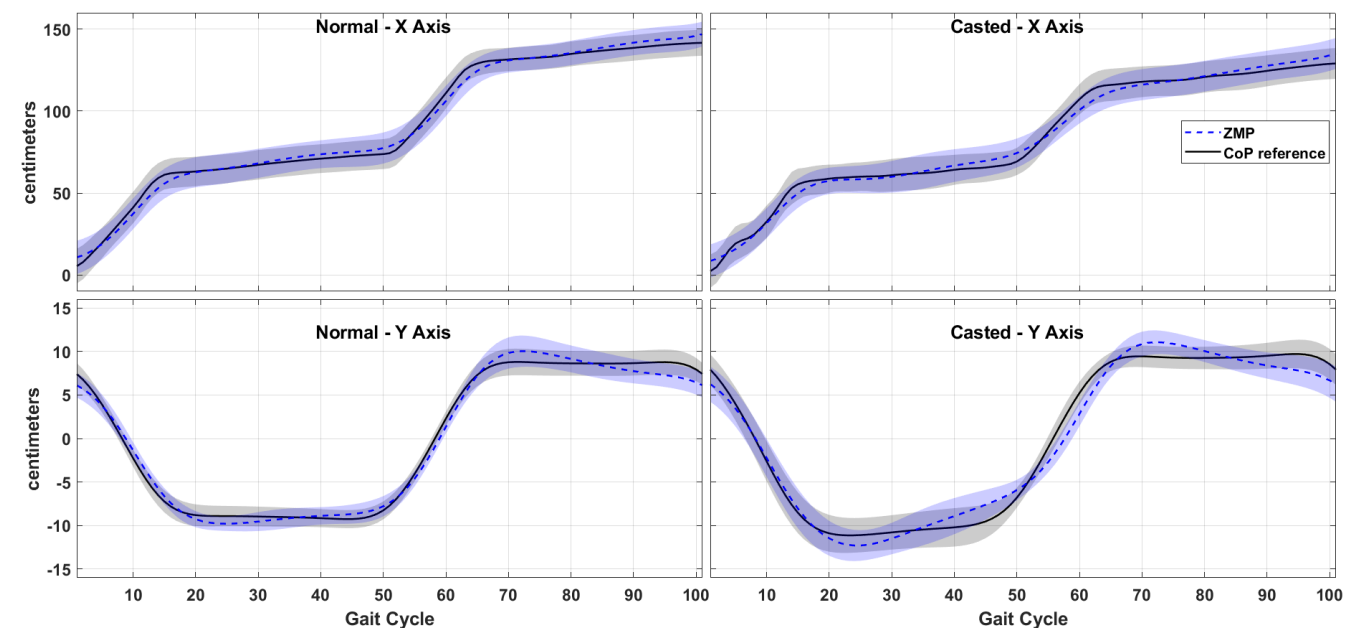

Fig. 2. Comparison of the mean trajectories of the zero moment point (ZMP) in dashed line and centre of pressure (CoP) in solid line for a normalised gait cycle. The cycle begins with right heel strike and ends with the subsequent right heel strike. The cycle is the average cycle for all subjects. The shaded regions show the standard deviation. The left column shows the normal condition and the right column shows the casted condition. The first row shows the trajectory in the $\mathrm{X}$ axis and the bottom row shows the trajectory in the $\mathrm{Y}$ axis.

differences are statistically significant using a two tailed ttest. Following this, we plot the trajectories of the interesting parameters in our example (Section II-E) with the IMUs.

\section{RESULTS}

Fig. 2 shows the normalised trajectories of ZMP and $\mathrm{COP}$ in the $\mathrm{X}$ and $\mathrm{Y}$ axes for both conditions: normal and casted. The graph shows the normalised gait cycle averaged over all subjects. The shaded regions show the standard deviation of the trajectories. The first column denotes the normal condition and the second column denotes the casted condition. Each row corresponds to one axis in the global frame. The mean absolute RMS of the differences between the ZMP and CoP over the complete cycle is shown in table I for both conditions. No statistically significant difference was found between the two variables. In table II, we compare the mean RMS of the distance between the ZMP and CoP across the gait cycle normalised by foot length $(\beta \%)$ with that of Herr et al [12]. Further, in table III, the mean RMS of the differences between the $\operatorname{pos}_{a x, r}$ and $\operatorname{pos}_{a x, l}$ and respective foot positions from GRAIL is shown for normal and casted walking conditions. It was found that only $\operatorname{pos}_{Y, r}$ during casted walking was not significantly different from the respective reference foot positions.

Finally, in Fig. 3, we see the trajectories of interesting parameters from a top-down view. The two triangles denote the starting foot positions. Both feet start at $0 \mathrm{~m}$ in the walking direction. The left foot starts at $0.2 \mathrm{~m}$ along the $\mathrm{Y}$ axis. The red dotted line represents the true CoM trajectory estimated from the ForceShoes ${ }^{\mathrm{TM}}$ [9] from start to end of $10 \mathrm{~m}$. The dark green dotted line is the trajectory of the $p_{0 s}^{w k} a_{a x, l}$, and the blue dotted line is the pos $s_{a x, r}^{w k}$ trajectory. These are estimated from the sensor fusion filter of Weenk et al [10]. They can be clearly seen to drift over time as they do not have information about relative distances. The intermittent solid lines denote the foot positions estimated from the CoM using ZMP assumption. Here, the yellow lines denote the $\operatorname{pos}_{a x, l}^{z m}$, measured at $s r$ and solid green lines denote the $\operatorname{pos}_{a x, r}^{z m}$, measured at $s l$.

TABLE I

MEAN RMS OF THE DIFFERENCES BETWEEN ZERO MOMENT POINT AND CENTRE OF PRESSURE OVER A GAIT CYCLE

\begin{tabular}{|c|c|c|}
\hline- & X Axis & Y Axis \\
\hline Normal $(\mathrm{cm})$ & $2.8 \pm 0.3$ & $0.8 \pm 0.2$ \\
\hline Casted $(\mathrm{cm})$ & $3.6 \pm 0.4$ & $1.4 \pm 0.3$ \\
\hline
\end{tabular}

TABLE II

MEAN DISTANCE BETWEEN ZERO MOMENT POINT AND CENTRE OF PRESSURE ACROSS GAIT CYCLE NORMALISED BY FOOT LENGTH $(\beta \%)$

\begin{tabular}{|c|c|c|}
\hline- & Herr et al. [12] & This study \\
\hline Normal (\%) & $14 \pm 2$ & $10.5 \pm 1.2$ \\
\hline Casted (\%) & - & $13.5 \pm 1.5$ \\
\hline
\end{tabular}

TABLE III

MEAN RMS OF DIFFERENCE BETWEEN pos $_{a x}$ AND REFERENCE FOOT POSITIONS AT RESPECTIVE INSTANCES. $\left({ }^{*}: p<0.05\right)$

\begin{tabular}{|c|c|c|c|c|}
\hline- & $\operatorname{pos}_{X, l}$ & $\operatorname{pos}_{Y, l}$ & $\operatorname{pos}_{X, r}$ & $\operatorname{pos}_{Y, r}$ \\
\hline Normal $(\mathrm{cm})$ & $9.5 \pm 0.8^{*}$ & $1.3 \pm 0.3^{*}$ & $9.3 \pm 0.6^{*}$ & $1.9 \pm 0.5^{*}$ \\
\hline Casted $(\mathrm{cm})$ & $8.9 \pm 1.4^{*}$ & $1.5 \pm 0.4^{*}$ & $12.8 \pm 1.8^{*}$ & $2.8 \pm 0.9$ \\
\hline
\end{tabular}

\section{DISCUSSION}

In practice, (3) is not valid. The moments around the CoM oscillate around zero [12, Fig. 3]. Upper body angular rotations also cause moments around the CoM. This is a missing component in (3). However, here we look at how 


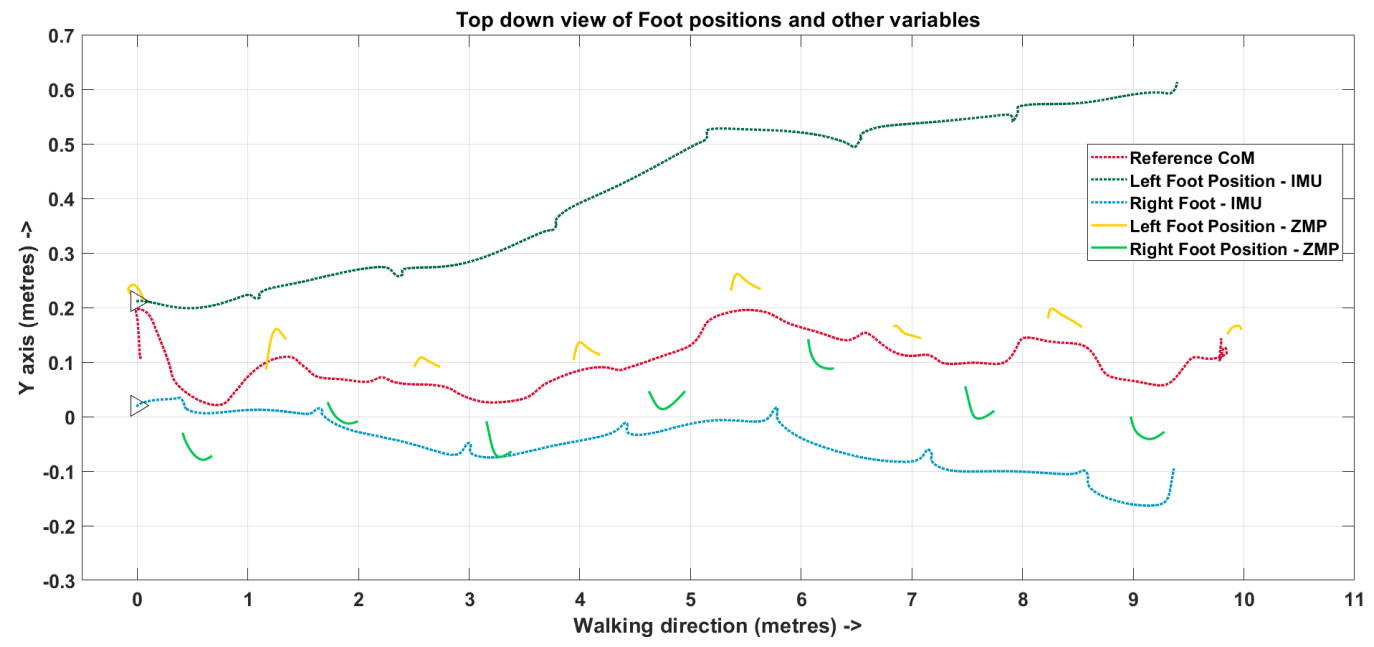

Fig. 3. Different trajectories from the example with IMUs in Section II-E are plotted. The dark green dotted line and blue dotted lines are the left and right foot trajectories respectively, estimated from the algorithm of Weenk et al [10] from the foot IMUs. The red dotted line is the reference centre of mass $(\mathrm{CoM})$ estimated by the ForceShoe ${ }^{\mathrm{TM}}$. The solid yellow lines are possible estimates for left foot during right swing phase, and solid green lines are possible estimates for the right foot during left swing phase. These are estimated using the CoM and zero moment point assumptions. The triangles denote the starting position of the two feet.

the ZMP and CoP agree, during straight walking, where the moments around the CoM may be really small.

Fig. 2 shows that there is close overlap between the trajectories of ZMP and CoP for the normalised gait cycle. The gait cycle begins with right heel strike and we can see the transition of the CoP from left to the right foot. The CoP falls completely under the right foot around $15 \%$ of the gait cycle. Following the left swing phase, we notice the left heel strike around $50 \%$ of the gait cycle, as the CoP starts to move towards the left foot. The trajectory continues to the next right heel strike which is the end of the gait cycle. Looking closer, we observe that the standard deviation of the trajectories (both ZMP and CoP) are smaller during the transition from one foot to the other. In both normal and casted conditions, the trajectory of ZMP is closer to the CoP in the $\mathrm{Y}$ axis during these transition (double stance) phases, when compared to the swing phases. This could suggest that the moments around the CoM are smaller during double stance phase, thereby showing lower differences in the ZMP and CoP trajectories during these instances.

Table I shows these differences as mean RMS of the differences between ZMP and CoP over the whole gait cycle. It is seen that the casting increases the error margins of the differences. The influence of casting on asymmetry of gait was verified by studying the step length. It was found that there were significant differences in the step lengths on the restricted foot before and after casting. Casting therefore, could induce asymmetry, causing increased rotation of the upper body to compensate for the change in walking pattern, and therefore, we see the differences in Table I. Table II shows the mean distance between ZMP and CoP across the gait cycle, normalised by foot length of the subjects. The lower errors in this study could be due to the use of the method of Schepers et al. [9], for estimating CoM, as both the low and high frequency information are present. However, this inference should be tested.

Table III shows the differences between foot positions estimated from CoM using ZMP assumptions and true foot positions from GRAIL system. The larger errors can be explained by the fact that we are actually comparing ZMP estimates from CoM with foot positions. We assume ZMP to lie close to CoP for each foot, and in turn, assume differences between $\mathrm{CoP}$ and foot positions to be trivial. Therefore, the table III shows the error margins associated with these assumptions. Looking closer, the table shows that the error margins are around $9.5 \mathrm{~cm}$ in $\mathrm{X}$ axis, and about $1.6 \mathrm{~cm}$ in the $\mathrm{Y}$ axis, for the normal walking condition. They show larger deviations in case of casting. These margins give us an idea of the feasibility of using ZMP based assumptions for estimating foot positions from CoM.

In Fig. 3, we see the different trajectories of interest, during an over-ground walking situation. Here, we see that the feet drift away from each other as there is no relative distance information. Therefore, we could use the estimates of right and left feet from CoM and ZMP to reduce this drift. Fig. 3 shows these estimates, $\operatorname{pos}_{a x, r}^{z m}$, and $p o s_{a x, l}^{z m}$, (denoted as solid green and yellow lines) oscillating on either side of the CoM. These lines are present only at the respective gait phases, either during left swing, or during right swing. This information could be used as a measurement update in a sensor fusion filter, at the right instances. As these filters, such as Kalman Filter, work with uncertainty margins, the error margins (shown in table III) could be accommodated for.

Thus, this shows that a minimal sensing system could consist of three IMUs; one on each foot, and one at the pelvis. The foot IMUs could track the movement of the feet in 3D. Measurement updates such as zero velocity update will minimise the drift in the $\mathrm{X}$ and $\mathrm{Z}$ directions [10]. The CoM can be tracked using a pelvis IMU [11]. The ZMP 
assumptions shown in this study could be used during left swing to estimate relative position of CoM relative to right foot. Additionally, during right swing phase, we can estimate CoM relative to left foot. If we fuse all this information, we can estimate the relative positions between the two feet during subsequent stance phases. This removes the need for full body sensing [2], or an inter-foot distance sensor [10], [13]. However, these assumptions need to be validated using a separate study.

Equation (5) also requires knowledge of the height of the CoM and forces in 3D. CoM height can be measured by the pelvis IMU, with appropriate measurement updates [11]. Estimating forces in 3D could be solved by either using pressure insoles, or a sensor fusion approach that measures the rotations of the pelvis in 3D. If we assume that the body is only in contact with the ground, then the accelerations of the pelvis could be similar to the accelerations at the CoM. This is simply the specific ground reaction forces in $3 \mathrm{D}$.

The current method assumes that the CoM position is used as a reference, and the estimates of the two feet could be corrected based on (6) and (7). An alternative method is to assume the right foot to be a reference point and then estimate the CoM, and subsequently, left foot position.

\section{A. Limitations and Conclusions}

The measurements were done on a treadmill which result in repetitive gait patterns. These are suitable for analysis, although, these patterns are not present in daily life. It is interesting to study the validity of ZMP assumptions during overground walking, and while performing tasks of daily life, and also asymmetric gait patterns. These evaluations would provide some indication about its use in minimal sensing of gait in a remote setting, or people with impaired gait. Asymmetrical walking may require the use of a sternum IMU to measure rotations of the upper body, to account for possible additional moments during walking.

The errors in table III are majorly present as we compare ZMP with reference foot positions. Therefore, a possible solution could be to measure CoP during walking, as they show lower errors with ZMP, as can be seen in table I. In an ambulatory sensing setup, these errors could be solved by using a pressure insole to measure $\mathrm{CoP}$ providing more accurate relative distances between CoM and either foot.

This study shows possible applications of using ZMP assumptions to reduce the lateral drift during minimal sensing of gait using IMUs. The next step is indeed to build a sensor fusion algorithm (with a setup similar to II-E) that can implement these updates iteratively. It is advised that the assumptions are studied for different walking patterns.

\section{REFERENCES}

[1] S. M. Bruijn, O. G. Meijer, P. J. Beek, and J. H. van Dieën, "Assessing the stability of human locomotion: a review of current measures." Journal of the Royal Society, Interface / the Royal Society, vol. 10, no. 83, p. $20120999,2013$.

[2] D. Roetenberg, H. Luinge, and P. Slycke, "Xsens MVN : Full 6DOF Human Motion Tracking Xsens MVN : Full 6DOF Human Motion Tracking Using Miniature Inertial Sensors, Tech. Rep. 3, 2009.
[3] A. Karatsidis, G. Bellusci, H. Schepers, M. de Zee, M. Andersen, and P. Veltink, "Estimation of Ground Reaction Forces and Moments During Gait Using Only Inertial Motion Capture," Sensors, vol. 17, no. 1, p. 75, 2016.

[4] F. J. Wouda, M. Giuberti, G. Bellusci, E. Maartens, J. Reenalda, B.J. F. van Beijnum, and P. H. Veltink, "Estimation of Vertical Ground Reaction Forces and Sagittal Knee Kinematics During Running Using Three Inertial Sensors," Frontiers in Physiology, vol. 9, no. March, pp. 1-14, 2018.

[5] G. Pacini Panebianco, M. C. Bisi, R. Stagni, and S. Fantozzi, "Analysis of the performance of 17 algorithms from a systematic review: Influence of sensor position, analysed variable and computational approach in gait timing estimation from IMU measurements," Gait and Posture, vol. 66, no. August, pp. 76-82, 2018.

[6] H. Zhao, Z. Wang, S. Qiu, Y. Shen, L. Zhang, K. Tang, and G. Fortino, "Heading Drift Reduction for Foot-Mounted Inertial Navigation System via Multi-Sensor Fusion and Dual-Gait Analysis," IEEE Sensors Journal, vol. PP, no. 8, p. 1, 2018.

[7] I. Skog, J. O. Nilsson, D. Zachariah, and P. Handel, "Fusing the information from two navigation systems using an upper bound on their maximum spatial separation," 2012 International Conference on Indoor Positioning and Indoor Navigation, IPIN 2012 - Conference Proceedings, no. November, pp. 13-15, 2012.

[8] K. Sorao, T. Murakami, and K. Ohnishi, "A unified approach to ZMP and gravity center control in biped dynamic stable walking," in Proceedings of IEEE/ASME International Conference on Advanced Intelligent Mechatronics. IEEE, p. 112.

[9] H. Schepers, E. van Asseldonk, J. Buurke, and P. Veltink, "Ambulatory Estimation of Center of Mass Displacement During Walking," IEEE Transactions on Biomedical Engineering, vol. 56, no. 4, pp. 11891195, 2009.

[10] D. Weenk, D. Roetenberg, B.-J. J. F. van Beijnum, H. J. Hermens, and P. H. Veltink, "Ambulatory Estimation of Relative Foot Positions by Fusing Ultrasound and Inertial Sensor Data." IEEE transactions on neural systems and rehabilitation engineering, vol. 23, no. 5, pp. 817-26, sep 2015.

[11] M. J. Floor-Westerdijk, H. M. Schepers, P. H. Veltink, E. H. F. Van Asseldonk, and J. H. Buurke, "Use of inertial sensors for ambulatory assessment of center-of-mass displacements during walking," IEEE Transactions on Biomedical Engineering, vol. 59, no. 7, pp. 20802084, 2012.

[12] H. Herr and M. Popovic, "Angular momentum in human walking," Journal of Experimental Biology, vol. 211, no. 4, pp. 467-481, 2008.

[13] S. Bertuletti, A. Cereatti, M. Caldara, and U. D. Croce, "A proximity sensor for the measurement of the inter-foot distance in static and dynamic tasks," Gait \& Posture, vol. 49, no. 2016, p. S15, 2016. 